\title{
A Compact Two-Dimensional Grating Coupler Used as a Polarization Splitter
}

\author{
Dirk Taillaert, Student Member, IEEE, Harold Chong, Peter I. Borel, Lars H. Frandsen, \\ Richard M. De La Rue, Fellow, IEEE, and Roel Baets, Senior Member, IEEE
}

\begin{abstract}
We demonstrate a novel polarization splitter based on a two-dimensional grating etched in a silicon-on-insulator waveguide. The device couples orthogonal modes from a single-mode optical fiber into identical modes of two planar ridge waveguides. The extinction ratio is better than $18 \mathrm{~dB}$ in the wavelength range of $1530-1560 \mathrm{~nm}$ and the coupling efficiency is approximately $20 \%$.

The device is very compact and couples light only to transverse-electric modes of the planar waveguides. Therefore, it may be used in a polarization diversity configuration to implement a polarization insensitive photonic integrated circuit based on photonic crystal waveguides.
\end{abstract}

Index Terms-Grating coupler, integrated optics, polarization, silicon-on-insulator (SOI).

\section{INTRODUCTION}

$\mathbf{P}$ HOTONIC crystal waveguides and photonic wires show great potential for high-density integrated circuits. Ultracompact waveguides in silicon-on-insulator (SOI) with relatively low losses have been demonstrated [1], [2]. However, the strong polarization dependence of these waveguides is a major disadvantage and limits the potential use in optical fiber communication networks. Also it is difficult to achieve a high coupling efficiency to fiber because of the huge mismatch in spotsize between these waveguides and a fiber.

Grating couplers can be used to couple top illuminating light into submicrometer-thin SOI waveguides [3]. Grating couplers using one-dimensional gratings (grooves) have extensively been studied, both theoretically and experimentally (see, e.g., [3] and [4]). It has also been demonstrated that a grating coupler can be used to butt-couple light between a vertical single-mode fiber and these waveguides [4]. In this letter, we demonstrate, for the first time, a grating coupler that also functions as a polarization splitter.

\section{CONCEPT}

It is well known that a single-mode fiber can carry two orthogonal linearly polarized modes [5]. When launching light into the

Manuscript received April 1, 2003; revised May 28, 2003. This work was supported in part by the European Union under the IST Project PICCO, and in part by the Belgian IAP PHOTON Project.

D. Taillaert and R. Baets are with the Department of Information Technology (INTEC), Ghent University_IMEC, B-9000 Gent, Belgium (e-mail: Dirk.Taillaert@intec.rug.ac.be).

H. Chong and R. M. De La Rue are with the Department of Electrical Engineering, Glasgow University, G128LT Glasgow, U.K.

P. I. Borel and L. H. Frandsen are with Research Center COM, Technical University of Denmark, DK-2800 Kgs. Lyngby, Denmark.

Digital Object Identifier 10.1109/LPT.2003.816671

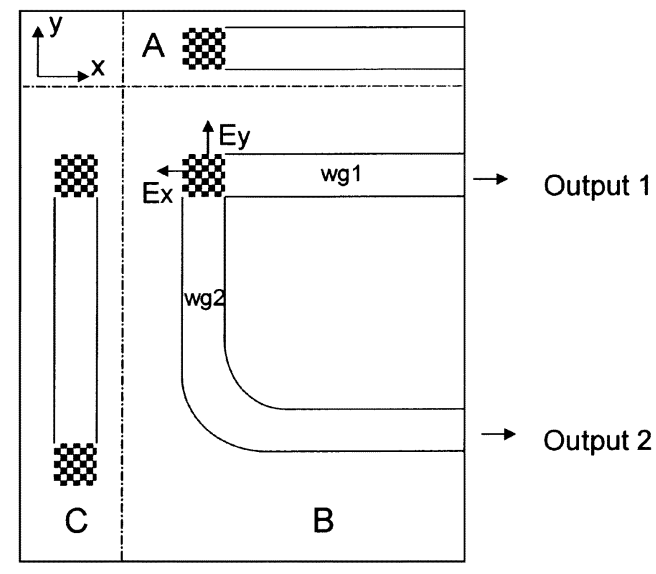

Fig. 1. Schematic top view of (A) grating coupler in a single waveguide, (B) grating coupler used as polarization splitter, and (C) waveguide with a grating coupler at both ends.

fiber, the total field will be in an elliptical state of polarization. Also the state of polarization changes during propagation in a real fiber due to polarization mode dispersion.

Consider a grating coupler consisting of a two-dimensional (2-D) grating etched in a ridge waveguide [Fig. 1(A)]. In general, the coupling efficiency from fiber to waveguide is polarization dependent. For the waveguides and gratings we use, the coupling efficiency for transverse electric (TE) is much larger than the coupling efficiency for transverse magnetic (TM). The coupling from fiber to waveguide will be maximized if the input is linearly polarized in the $y$ direction. This polarization dependence is mainly caused by the difference in effective index between the fundamental TE and TM waveguide mode: The grating couples both modes out toward different angles, and if the difference is sufficient, only one of them can couple efficiently to a fiber.

The structure of Fig. 1(B) consists of two ridge waveguides that are orthogonal with regard to each other. The 2-D grating is etched into the crossing of the waveguides and is invariant for a $90^{\circ}$ rotation. Light from the fiber with linear polarization with electric field $E y$ will couple to Waveguide 1 and the other polarization with electric field $E x$ to Waveguide 2. In general, elliptically polarized light from a single-mode fiber will couple both to Waveguide 1 and 2 . The coupling ratio will depend on the state of polarization, but the total coupling to Waveguide 1 and Waveguide 2 will be independent of it. The $90^{\circ}$ bend in Waveguide 2 is only used for practical reasons (to have the two outputs at the same cleaved facet). We have described the working principle as input coupler, but the device can also be used as output 


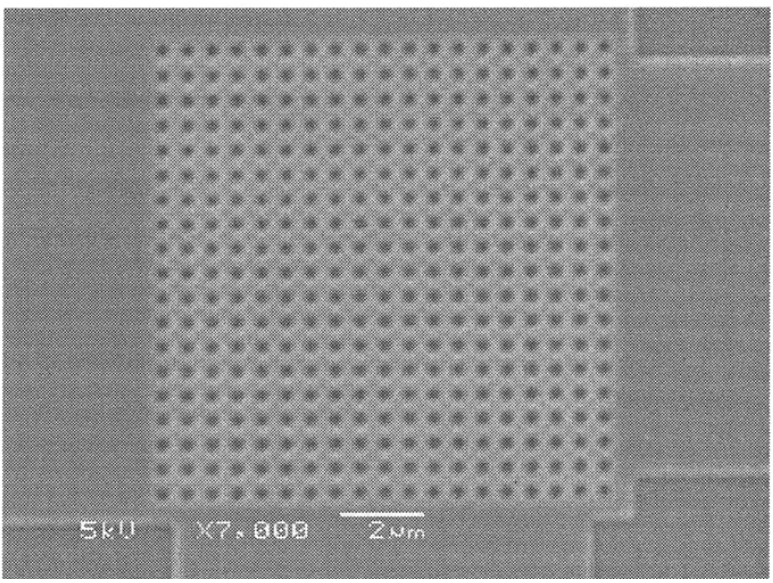

Fig. 2. Scanning electron micrograph top view of a 2-D grating coupler. The lattice period of the square array of holes is $580 \mathrm{~nm}$.

coupler. The coupling efficiency from waveguide to fiber is the same as from fiber to waveguide when considering the coupling from one mode to another mode because of reciprocity.

The coupler is designed for normal incidence to achieve a good extinction ratio. With normal incidence and a symmetric grating the maximum achievable coupling efficiency is $50 \%$. Therefore, an asymmetric or blazed grating will be needed in a practical device.

There is a very important difference between conventional polarization splitters described in the literature [6] and our splitter. In a conventional splitter, the input light is split in a TE and TM waveguide mode. In our device, the light from a fiber is split in two TE waveguide modes. Because in the two waveguides the same polarization (TE) is used, the device can be useful to make polarization insensitive photonic integrated circuits, using waveguides which are very strongly polarization sensitive [7].

\section{EXPERIMENTAL RESULTS}

\section{A. Fabricated Structures}

The structures were fabricated on SOI wafers with a top silicon film thickness of $220 \mathrm{~nm}$ and a buried $\mathrm{SiO}_{2}$ layer thickness of $1 \mu \mathrm{m}$. Gratings and waveguides were defined using electron-beam lithography and reactive ion etching. A top view is shown in Fig. 2. The grating is a square array of holes, the etch depth is $90 \mathrm{~nm}$, and the lattice period $580 \mathrm{~nm}$. Different hole diameters were tried and the optimum hole diameter is found to be $300 \mathrm{~nm}$. The ridge waveguides are $10 \mu \mathrm{m}$ wide. The effective index of the fundamental waveguide mode is 2.83 for TE and 1.89 for TM polarization. The coupler size approximately matches the spot-size of the fiber but the coupler size and coupling length are not yet optimized. A $90^{\circ}$ bend with a radius of $530 \mu \mathrm{m}$ is inserted in one of the waveguides to be able to detect both outputs at one cleaved facet. The length of Waveguide 1 is $850 \mu \mathrm{m}$.

\section{B. Extinction Ratio}

We used the structures from Fig. 1(B) to measure the extinction ratio and check the polarization of the light in the waveguides. A vertical single-mode fiber couples light from a tunable

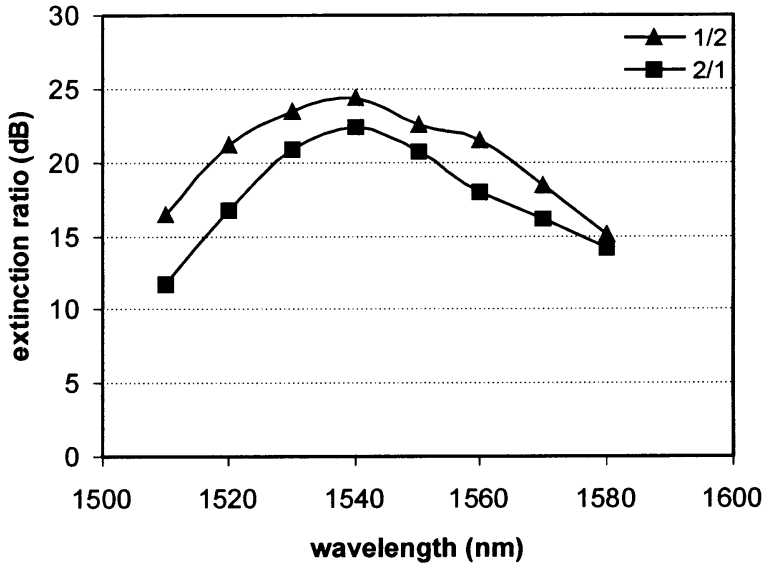

Fig. 3. Measurement of the extinction ratio between Output 1 and 2. The difference between the two curves is caused by losses in the $90^{\circ}$ bend in Waveguide 2 .

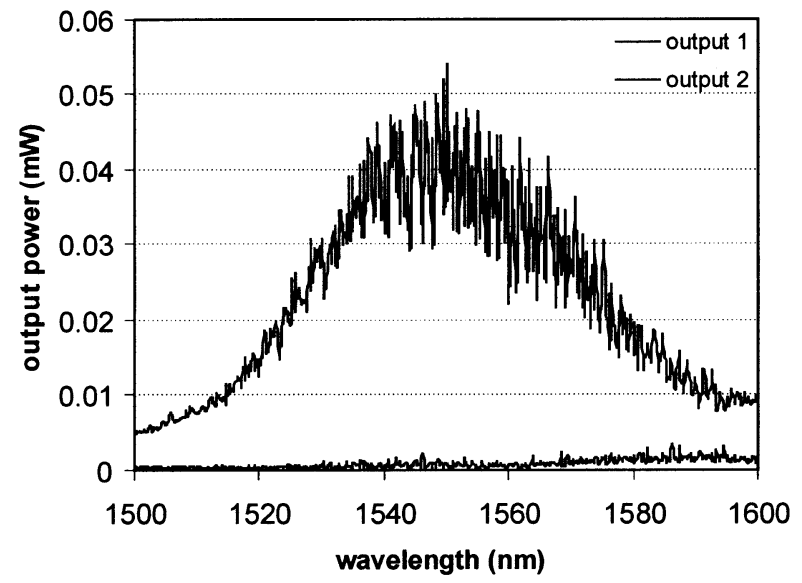

Fig. 4. Measurement of output power versus wavelength. The polarization of the input is changed to maximize Output 1 at 1550 -nm wavelength before this measurement.

laser into the waveguides via the grating. We detect the output power of Waveguides 1 and 2 at the cleaved facet using a $40 \times$ objective and a power detector.

The first step is alignment of the fiber. To make sure that the center of the fiber is aligned with the center of the grating, the $y$ position is optimized using Output 1 and the $x$ position using Output 2. The height of the fiber above the sample is approximately $10 \mu \mathrm{m}$. The beam size on the coupler is estimated to be $10.6 \mu \mathrm{m}\left(1 / e^{2}\right.$ diameter of the power distribution).

After the initial alignment, the position of the fiber is fixed and only the polarization and wavelength are changed during the further measurements. The polarization of the input light is changed using a Lefevre-type polarization controller to maximize Output 1 or Ouput 2 . Then the extinction ratio between the two outputs is measured for both cases. The extinction ratio is shown in Fig. 3 for different wavelengths. The extinction ratio is better than $18 \mathrm{~dB}$ in the wavelength range of $1530-1560 \mathrm{~nm}$. The difference between the two cases is caused by radiation losses in the $90^{\circ}$ bend in Waveguide 2. We have also verified the polarization of the light in the waveguides. The output power versus wavelength for the case when Output 1 is maximized is shown in Fig. 4. The fringes in these curves are caused by 


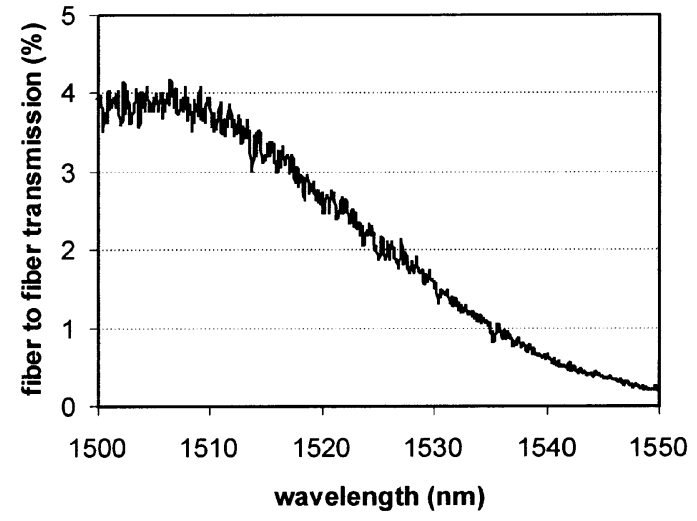

Fig. 5. Measurement of the power transmission from fiber-to-fiber on a waveguide with a grating coupler at both ends.

a cavity between the grating (second-order reflection) and a cleaved facet. The fringe spacing is approximately $0.5 \mathrm{~nm}$, this is consistent with the waveguide length and effective index. The extinction ratio we have measured is probably limited by our measurement setup, because the extinction ratio of the input light, measured with a rotatable polarizer, is only $25 \mathrm{~dB}$.

\section{Coupling Efficiency}

It is not straightforward to determine the coupling efficiency from the power detected at the cleaved facet because of the facet reflectivity and the high numerical aperture of the waveguides. Therefore, we estimate the coupling efficiency from a transmission measurement from fiber to waveguide to fiber on a structure consisting of a waveguide with a grating coupler at both ends [Fig. 1(C)]. In this measurement, the fibers are tilted slightly to shift the wavelength out of the second-order grating reflection peak and, thus, avoid cavity effects between the two gratings. As shown in Fig. 5, the fringes are smaller and the fiber-to-fiber transmission is $4 \%$ for TE polarization. This transmission includes two couplers, so the coupling efficiency is $20 \%$ if we take into account that the coupling from fiber to waveguide is the same as from waveguide to fiber.

\section{CONCLUSION}

We have proposed and demonstrated a 2-D grating coupler used as a polarization splitter. The current device is a proof-ofprinciple, but two issues need to be solved before it can be used in practical applications: the coupling efficiency should be increased and the grating reflection reduced. The efficiency can be improved by using an optimized grating design and SOI wafers with a buried reflector [8]. The reflection may be reduced by using a properly designed asymmetric grating.

\section{REFERENCES}

[1] M. Notomi, A. Shinya, K. Yamada, J. Takahashi, C. Takahashi, and I. Yokohama, "Structural tuning of guiding modes of line-defect waveguides of silicon-on-insulator photonic crystal slabs," IEEE J. Quantum Electron., vol. 38, pp. 736-742, July 2002.

[2] K. L. Lee, D. R. Lim, L. C. Kimerling, J. Shin, and F. Cerrina, "Fabrication of ultralow-loss $\mathrm{Si} / \mathrm{SiO}_{2}$ waveguides by roughness reduction," Opt. Lett., vol. 26, pp. 1888-1890, Dec. 2001.

[3] R. M. Emmons and D. G. Hall, "Buried-oxide silicon-on-insulator structures: Waveguide grating couplers," IEEE J. Quantum Electron., vol. 28, pp. 164-175, Jan 1992.

[4] D. Taillaert, W. Bogaerts, P. Bienstman, T. F. Krauss, P. Van Daele, I. Moerman, S. Verstuyft, K. De Mesel, and R. Baets, "An out-of-plane grating coupler for efficient butt-coupling between compact planar waveguides and single-mode fibers," IEEE J. Quantum Electron., vol. 38, pp. 949-955, July 2002.

[5] E. G. Neumannn, Single-Mode Fibers - Fundamentals. Berlin, Germany: Springer-Verlag, 1988, ch. 5.

[6] J. Van der Tol, J. W. Pedersen, E. G. Metaal, J. Van Gaalen, Y. S. Oei, and F. H. Groen, "A short polarization splitter without metal overlays on InGaAsP-InP,” IEEE Photon. Technol. Lett., vol. 9, pp. 209-211, Feb. 1997.

[7] D. Taillaert and R. Baets, "Photonic Crystal Based Fiber-to-Waveguide Coupler for Polarization Independent Photonic Integrated Circuits," patent pending.

[8] M. K. Emsley, O. Dosunmu, and M. S. Unlu, "Silicon substrates with buried distributed Bragg reflectors for resonant cavity enhanced opto-electronics," IEEE J. Select. Topics Quantum Electron., vol. 8, pp. 948-955, July/Aug. 2002. 